\title{
A social and physical stressor increases later punishment in healthy males
}

Short title: Situational stress increases punishment

Yacila I. Deza-Araujo ${ }^{1,2}$, Ulrike Rimmele ${ }^{1,2,3,4}$, Lucien Gyger ${ }^{6}$, Cora Aguirre ${ }^{4}$, David Sander ${ }^{1,7}$, Olga M. Klimecki ${ }^{1,2,8 *}$

${ }^{1}$ Swiss Center for Affective Sciences, University of Geneva, Geneva, Switzerland.

${ }^{2}$ Laboratory for Behavioral Neurology and Imaging of Cognition, Department of Neuroscience, Medical School, University of Geneva, Geneva, Switzerland.

${ }^{3}$ Department of Basic Neurosciences, University of Geneva Switzerland.

${ }^{4}$ Faculty of Psychology and Educational Sciences, University of Geneva, Switzerland

${ }^{5}$ Center for Interdisciplinary Study of Gerontology and Vulnerability (CIGEV), University of Geneva, Switzerland.

${ }^{6}$ Université Grenoble Alpes, CNRS, LPNC UMR 5105, Grenoble, France

${ }^{7}$ Laboratory for the Study of Emotion Elicitation and Expression, Department of Psychology, University of Geneva, Geneva, Switzerland.

${ }^{8}$ Clinical Psychology and Behavioral Neuroscience, Faculty of Psychology, Technische Universität Dresden, Dresden, Germany.

*Corresponding author:

E-mail address: olga.klimecki@unige.ch (OMK) 


\section{Abstract}

1 Existing literature suggests that stress in early life can influence or trigger later aggressive and

2 punishment behaviors during social interactions. However, only few human studies have addressed this

3 link in controlled experimental settings. Here, we assessed the impact of biological and behavioral

4 markers of stress on economic and social interactions in healthy men using a between group design. The

5 Socially Evaluated Cold Pressor Test (i.e., supervised hand immersion into cold water) was applied

6 to one group of participants $(n=19)$, while a second group underwent the Warm Water Test $(n=21)$.

7 After the stressor or control procedure, all participants played the Inequality Game, a paradigm in

8 which they could engage in punishment, cooperative, or aggressive behaviors towards a fair and an

9 unfair counterpart player. Compared to the control condition, participants in the stress condition engaged in more punishment behaviors towards the unfair player and less cooperative behaviors towards both

11 players. Critically, higher levels of cortisol in the stress group were associated with more punishment

12 behaviors towards the unfair player. In contrast, aggressive behavior did not differ between participants in the stress vs. control condition. Overall, our findings showed that situationally induced stress might facilitate punishment behaviors in provoking situations. Further research should elucidate the role of inter-individual variables that may encourage or prevent stress-related punishment in social contexts. 


\section{Introduction}

In human interactions, the behaviors of punishment and aggression are triggered by complex psychobiological processes that are typically mobilized when one perceives a hostile or unfair situation $(1,2)$. Conceptually, punishment occurs when an unpleasant stimulus is imposed on an individual in order to discourage his or her current behaviour (3). Punishment can have a positive connotation when it is aimed to discourage norm violations (i.e. altruistic punishment $(4,5)$ ). Furthermore, punishment can be used to provide relief and satisfaction after interpersonal conflicts (e.g. to take revenge after having been treated unfairly). Aggression, on the other hand, usually has a negative connotation. It is defined as an intense behaviour aimed at inflicting pain or physical damage on another individual (6). Due to the often undesirable consequences of aggression and active punishment in social contexts (7) several studies have been conducted to elucidate the manifestations and causes of these behaviors in different environments $(8,9)$. Yet, the psychological and biological mechanisms underlying active punishment and aggression in humans merit a more detailed investigation.

Existing longitudinal human studies point to the possibility that one factor, namely stress, may trigger aggressive responses, especially when the organism has repeatedly and unsuccessfully tried to cope with a given situation (10). A classic example is the presence of early life stressors, which increase the risk of antisocial behaviors during adolescence and adulthood $(10,11)$. In a similar manner, sudden stressors in everyday life, such as traffic jams or bad news, can have immediate effects on negative moods and emotions, increasing the risk of violent outbursts (12-14). Conversely, few studies have suggested that acute stressors can actually promote prosocial behaviors and altruism in natural (15) and experimental $(16,17)$ settings. While the exact nature of these discrepancies remains a matter of debate (18), current research has shown that the so-called "tend-and-befriend" pattern under stress is highly influenced by the group affiliation (18). Hence, controlled experiments with outgroup members may help to disentangle the link between stress and later aggression and punishment in provoking situations.

On a psychological level, stress is typically elicited when an event is appraised as threatening to the physiological and psychological integrity of an individual who assesses his or her resources as insufficient to successfully cope with the stressful event and its consequences $(19,20)$. On a 
physiological level, stress is usually perceived as a general alarm reaction accompanied by the activation of a cascade of biological processes. These processes trigger the adrenal cortex to release cortisol into the bloodstream (21) and activate the sympathetic nervous system, resulting in increased arousal (22). These biological reactions support the "fight or flight" response, which may result in aggression in situations of high perceived provocation and weak coping strategies (13). Physiologically, arousal is characterized by an increase in central and peripheral levels of noradrenaline. This prepares the organism for rapid and energetic responses and likely interferes with higher cognitive functions, mediating reactive behaviors in potential situations of aggression $(6,23)$. This notion is further supported by studies showing that recurrent exposure to stressors impairs the functioning of the prefrontal cortex (24) and has deleterious effects on inhibitory control, thereby promoting a series of problematic behaviors such as compulsive reward-seeking behavior, relapse in substance addiction (20), or antisocial behaviors (25).

Animal experiments provided robust evidence for a link between stress and aggressive behaviors. For instance, Marquez and colleagues showed that early stress in rats (i.e., fear induction procedures) leads to indiscriminate aggressive responses, which are accentuated by epigenetic modifications in frontolimbic systems (26). In the same vein, Sandi and Haller (27) provided compelling evidence for a direct connection between early life stressors and higher rates of aggressive and antisocial behaviors in older years in both humans and animals. Contrasting animal experiments, human studies in controlled laboratory settings are scarce and often assess only subjective ratings and questionnaires. For example, Sprague and colleagues showed that self-reported measures of subjective experience of stress (e.g., health- or job- related) and feelings of state anger and hostility are related, particularly in participants with lower executive functions and lower socio-economic status (28). Similarly, Hennessy demonstrated that questionnaires relating commuter stress and aggression in the workplace were positively correlated, especially in male individuals (14). Another study showed that under higher levels of experimentally induced stress (measured with subjective and endocrine responses), participants with higher traits of aggression exhibited more approaching behaviors in an approach-avoidance task (29).

In contrast to the studies describing the link between stress and aggression in social contexts reported above (for review, see (27)), the specific link between stress and punishment behaviors is less known. 
In animals, this link is difficult to study, since punishment among animals societies is aimed to inflict discipline and maintain cooperation (30). Therefore, only human studies offer the opportunity to investigate how stress may modulate the presentation and intensity of both reactive aggression and active punishment. So far, only one human study that examined the link between stress and punishment, showed that participants were less generous and had higher propensity for altruistic punishment during the Ultimatum Game immediately after an experimental stress induction (31).

In the present study, we aimed to further investigate the impact of stress on both aggressive and punishment behaviors. To induce stress, we used the Socially Evaluated Cold Pressor Test - SECPT (32) in a group of participants, while a second group underwent a control condition with the Warm Water Test - WWT (32). Cooperative and competitive economic behaviors following the stressor were quantified using the Inequality Game - IG (33), a paradigm designed to investigate emotional and behavioral reactions to fair and unfair exchanges of feedback and economic choices. Along with previous findings showing that everyday stressors are related to higher rates of aggression in social environments $(14,28)$, we hypothesized that participants in the stress group will show more punishment behavior, more aggressive behavior and less cooperative behavior during the IG.

We included only male participants because previous research suggested sex differences in aggressive behaviour (34) and stress responses (35). Additionally, we analysed measures of salivary cortisol and alpha-amylase to investigate their association with aggression and punishment behaviors after the stress induction. Finally, questionnaire-based measures were used to assess behavioral variables that may intensify or moderate the presentation of aggression and punishment in both groups. 


\section{Methods}

\section{Participants}

90

91

92

93

Fifty-one healthy males were recruited from the Geneva area. Inclusion criteria were: between 18 and 50 years of age, non-smokers, French-speaking. Participants were asked to refrain from caffeine or juice intake at least two hours before the experiment and not to practice any sport or any demanding physical activity four hours before the experiment. They were randomly assigned to either the stress or the control condition. Two participants were excluded because they had participated in a similar study before. Nine participants were excluded because they did not believe that they were playing with a real player during the Inequality Game. The final sample consisted of 40 males (mean age $=27.1$ years, $\mathrm{SD}=7.66,21 \mathrm{in}$ the control group, 19 in the stress group). Participants provided informed written consent and were remunerated with $20 \mathrm{CHF}$ per hour. All procedures in this study were followed in accordance with relevant guidelines. The study was approved by the Research Ethics Committee of the Faculty of Psychology and Educational Sciences at the University of Geneva.

\section{Procedure}

After arriving at the laboratory, inclusion criteria were verified and participants provided informed consent. To avoid bias in their performance during the experiments the official title of the study was Study of the influence of somatosensory stimulation on decision-making processes. Every participant was told twice (in the general consent form and orally, before the stress induction) that they could interrupt the experiment at any moment without having to provide any justification.

\section{Neuroendocrine Stress Measures}

All experimental sessions were carried out after noon to control for the effects of the circadian rhythm on the secretion of cortisol and alpha-amylase $(36,37)$. Salivary samples were collected using Salivette tubes (Salivette, Sarstedt, Nümbrecht, Germany). The samples were stored at $-20^{\circ} \mathrm{C}$, and then sent out to the Technische Universität Dresden, Germany for biochemical analysis. The first salivary sample was collected immediately after the participant had signed the informed consent (baseline) and the following samples were collected every 15 minutes throughout the duration of the whole experiment. We collected 
eight salivary cortisol samples over a period of approximately two hours to fully capture the stressinduced cortisol peak and recovery. Cortisol levels were determined using a commercially available chemiluminescence immunoassay (CLIA; IBL Hamburg, Germany) with intra- and interassay variabilities of less than $10 \%$. Cortisol levels were calculated and expressed in nmol/l. Alpha-amylase levels were determined using an enzyme kinetic method (38). Alpha-amylase activity was calculated and expressed in $\mathrm{U} / \mathrm{ml}$.

For statistical analysis, repeated measures ANOVAs were implemented with "group" (control vs. stress) as between-subject factor and "time" (eight sampling points) as within-subject factors. Results were considered significant at a $p$ value of $<0.05$.

\section{Questionnaires}

Before the stress induction procedure, we asked participants to fill in the socio-demographic questionnaire and three additional scales:

Interpersonal Reactivity Index (IRI (39), French version (40)). The IRI measures forms of empathy using four scales: Distress, Empathic Concern, Perspective Taking and Fantasy. The minimum score in each subscale is 7 and the maximum is 49 .

The Positive and Negative Affect Schedule (PANAS (41), French version (42)). The PANAS is a bidimensional mood inventory with 20 items. The Positive Affect scale measures the degree to which an individual feels enthusiastic, active, and alert. The Negative Affect scale is an index of subjective distress. Items scale from 1 ("Very slightly or not at all") to 5 ("Extremely").

Visual Analog Scales to measure the level of stress, pain and unpleasantness. Adapted from (32, 43), three visual analogue scales measured the level of stress, pain and unpleasant feelings with anchors at $0=$ "not at all" and $100=$ "extremely" before and after the stressor/control procedure.

\section{Stress induction procedure}

The Socially Evaluated Cold Pressor Test - SECPT, a stressor with a physical and social component, was applied for the stress group and the Warm Water Test - WWT for the control group (32). Both 
procedures were previously used to investigating the effects of stress on cue-triggered wanting (44).

Briefly, participants were asked to keep their non-dominant hand in cold water $\left(0-4^{\circ} \mathrm{C}\right)$ for an indefinite period of time. However, the test was ended by the experimenter after three minutes if the participant did not take out his hand before (mean time of immersion $=1 \mathrm{~min} 36 \mathrm{sec}$., $\mathrm{SD}=1 \mathrm{~min} 6 \mathrm{sec}$.). To add social stress, participants were videotaped during the entire stress induction procedure and were asked to look straight at the camera. The experimenter stood in front of the participant and looked at him during the entire process. For the control procedure (WWT), participants were instructed to keep their hand in warm water during three minutes. The water was at body temperature $\left(35-37^{\circ} \mathrm{C}\right)$, there was no video recording and the experimenter did not look at the participant during this procedure. After the SECPT or WWT, participants were asked to report their levels of stress, pain, and unpleasant feelings using the three visual analogue scales, for a second time.

\section{Inequality Game}

To assess social behavior, all participants played the Inequality Game $(33,45)$ after the stress or control procedure. In this computer task, participants interacted economically and through written feedback messages with two alleged other players - one player being fair, and the other being unfair. During each economic interaction, the participant and one of the other players made a common decision on a $2 \times 2$ payoff matrix that determines the payoff for both players (the participant and the other player. Fig. 1). The task had two phases, an anger provocation phase (phase A, low power for the participant, high power for the other players) and a behavioral response phase (phase B, high power for the participant, low power for the other players) in which we assessed participants' behavioral responses to the provocation from the previous phase. The player in high power controled the lines and chose first. The player in the low power condition controled the columns and chose second. The player in the high power condition influenced/decided up to $90 \%$ of the other person's gain in a given trial. Before the game started, participants were told that the computer will randomly select who plays first, although the IG always started with low power for the participants.

In total, all participants underwent each phase (low and high power), which had 24 economic choices and 12 feedback choices, which were equally split into interactions with the fair and unfair other. Payoff 
magnitudes ranged from 0 to $10 \mathrm{CHF}$ in each economic choice. Twelve of the 24 economic choices reflected situations in which the participant in high power could choose between a cooperative and a competitive allocation of money for the other (e.g., 9/0, 8/9, 9/8 or 8/1 CHF). As a control condition, the remaining 12 trials were divided in either a win/win (e.g., 9/10, 10/9, 10/10, 9/10 CHF) or a no win/ no win outcome (e.g., 1/1, 1/1, 0/0,0/1 CHF). During the phase A (low power for the participant) and the control condition, the choices of the fair and unfair other always served to maximize their own gains. The payoffs in the IG were largely independent, which means that the choice of high or low amounts for the other player did not affect the final payoff of the participants.

During the low power phase, the unfair player chose competitive economic outcomes (e.g., 8 CHF for him and 1 CHF for the participants) and derogatory feedback for all the participants ("You are not nice" three times and "You are annoying" three times), while the fair other player always chose cooperative economic outcomes (e.g., 8 CHF for him and 9 CHF for the participants) and nice feedback ("You are very nice" three times and "You are nice" three times). The next phase gave the participants the opportunity to behave cooperatively or competitively towards both the unfair and the fair player. Specifically, competitive economic choices (i.e., reduce the other's gains) and derogatory feedback towards the unfair other player can be interpreted as punishment behaviour (5), while cooperative economic choices and nice feedback towards the same participant can be interpreted as forgiveness behavior. Conversely, competitive economic choices and derogatory feedback towards the fair other player indicates aggressive behavior (46) and cooperative economic choices and nice feedback indicates prosocial behavior.

To quantify the dependent behavioral variable of interest resulting from either the cooperative or competitive behavior towards the other player (i.e. punishment and forgiveness towards the unfair player, and aggressive and prosocial behavior towards the fair player), we computed the difference between the number of cooperative (positive values) choices and the number of competitive (negative values) choices in the economic interactions from the high power phase towards the fair and unfair other separately (phase B). To quantify feedback behavior, we summed the weighted frequencies with which participants chose each of the four feedback sentences ("You are very nice" $=+2$, "You are nice" $=+1$, 
"You are not nice" $=-1$, and "You are annoying" $=-2$ ). Positive values reflect nice messages, while negative values reflect derogatory messages. These scores were calculated separately for both, the fair and unfair other.

To ensure the engagement of participants in the economic interactions, they were told that two of their choices during the IG would be randomly picked and paid at the end of the session and this gain could be up to $20 \mathrm{CHF}$. However, all participants received the same amount (i.e., $20 \mathrm{CHF}$ per hour) for fairness reasons, as agreed with the ethics commission of the Psychology Department of the University of Geneva. For statistical analyses, a repeated measures ANOVA was implemented with "group" (control vs. stress) as between-subject factor and "other" (fair vs. unfair) player as within-subject factors.

At the end of the IG, ratings of stress, pain, and unpleasant feelings were collected for a third time. Moreover, we asked participants to give their opinion about the other player. Participants who did not believe that the other players were real were excluded from the analyses.

Figure 1

Fig 1. Inequality Game (IG, Figure from (33). The figure displays a timeline trial for phase B (high power for the participant, low power for the other players). The participant controls the lines and chooses first (in this example Nicolas in green), therefore he has the opportunity to behave in a cooperative way (e.g., by choosing the upper row which contains more equal amounts) or in a competitive way by choosing the lower row, as indicated in the figure. The other player chooses second and controls the columns (here, Jean in blue). The final result is given by the intersection of both choices (in this case, 8 CHF for Nicolas and 1 CHF for Jean). Half of the economic interactions were followed by a feedback trial, in which participants in high power could choose between two positive and two negative phrases.

At the end of the experimental session, we conducted a structured debriefing with the participants in which we revealed the nature of the manipulation (i.e., the purpose of the stress induction, and the nature of the IG). After the debriefing, participants were asked to confirm their consent. In addition, all participants were notified that the video recording were erased. Fig. 2 shows a depiction of the study design. 


\section{Results}

\section{Baseline Questionnaires}

Fig 2. Study design. Participants were randomly assigned to the stress $(\mathrm{N}=19)$ or control $(\mathrm{N}=21)$ group. A total of eight salivary samples, every 15 minutes, were collected during the whole experimental session. Upon arrival, at T0, the first salivary sample was taken as a baseline measure of cortisol levels and alpha-amylase activity. Participants then filled in the Interpersonal Reactivity Index (IRI), the Positive and Negative Affect Schedule (PANAS) and provided subjective intensity ratings of stress, pain and unpleasant feelings. Another salivary sample was taken (T1) and then the Socially Evaluated Cold Pressor Test (SECPT) was applied to the stress group and the Warm Water Test (WWT) was applied to the control group. After another salivary sample (T2), participants provided subjective feelings of stress, pain, and unpleasantness and received the instruction of the Inequality Game (IG). We took another salivary sample before phase A of the IG (T3), before phase B of the IG (T4) and after the end of the IG (T5). Participants then provided ratings of stress, pain, and unpleasantness, and another salivary sample was taken (T6). Participants had a 15 minutes break to allow for cortisol levels to return to baseline (measured at T7). Participants were then thanked, debriefed and paid.

All behavioral analyses were conducted using IBM SPSS Statistics for Windows (release 25.0, IBM Corp., Armonk, IL, USA) and R Version 3.6.1 (https://www.r-project.org/). Significance was considered at an alpha level of 0.05 (two-tailed). Since sphericity assumptions were not met, Greenhouse-Geisser correction was used for all analyses.

Pertaining to the trait questionnaires measured at baseline (Table 1), independent $t$-tests revealed no group differences on the Positive and Negative Affect Schedule (for positive or negative affect) between the groups (all $p_{s} \geq 0.56$ ). Pertaining to the Interpersonal Reactivity Index, independent $t$-tests showed that the stress and control group did not differ on the subscales Perspective Taking, Empathic Distress or Fantasy $\left(t(38)<0.88\right.$; all $\left.p_{\mathrm{s}} \geq 0.39\right)$. However, despite the randomization procedure, the control group had higher levels of Empathic Concern than the stress group $(t(38)=3.07 ; p=0.004)$. 


\begin{tabular}{lllll} 
& $\begin{array}{l}\text { Control Group } \\
\text { Mean (SD) }\end{array}$ & $\begin{array}{l}\text { Stress Group } \\
\text { Mean (SD) }\end{array}$ & $t_{(38)}$ & $p$ \\
\hline IRI Distress & $23.38(7.19)$ & $22.84(9.2)$ & 0.21 & 0.84 \\
IRI Empathic Concern & $37.48(5.08)$ & $32.89(4.29)$ & 3.07 & $0.004^{*}$ \\
IRI Perspective Taking & $36(5.17)$ & $34.16(7.95)$ & 0.88 & 0.39 \\
IRI Fantasy & $29.14(5.23)$ & $28.58(6.64)$ & 0.3 & 0.77 \\
\hline PANAS Positive Affect & $30.76(7.06)$ & $29.74(4.58)$ & 0.54 & 0.59 \\
PANAS Negative Affect & $13.29(4.98)$ & $14.21(4.85)$ & 0.59 & 0.56 \\
\hline
\end{tabular}

$\mathrm{N}=40 . \mathrm{IRI}=$ Interpersonal Reactivity Index; PANAS $=$ The Positive and Negative Affect Schedule; SD $=$ Standard Deviation.

\section{Participants in the stress group reported higher levels of pain and unpleasantness, but no evidence} was found for higher levels of stress

In order to test whether there was an effect of "group" (stress vs. control) or "time" (before the stress/control induction, directly after it, and at the end of the experiment) on subjective levels of stress, pain, and unpleasant feelings, we conducted repeated measures ANOVAs for each of these variables. These analyses revealed that for self-reported stress, there was a significant main effect of time $(F(1.42$, $\left.53.98)=3.97 ; p=0.03, \eta_{P}^{2}=0.95\right)$, but no significant effect of $\operatorname{group}(F(1,38)=0.46 ; p=0.83)$ and no interaction effect (time $\mathrm{x}$ group, $F(1.42,53.98)=0.24 ; p=0.71$ ). Post-hoc tests revealed a marginal decrease in reported stress levels from the first to the second time point ( $p=0.05$; two-tailed), and no difference between the second and the third time point ( $p=0.15$; two-tailed), collapsed across both groups. Regarding the subjective ratings of pain, there was a significant main effect of time $(F(2,75.99)$ $\left.=11.30 ; p<0.001 ; \eta_{P}^{2}=0.23\right)$, a significant effect of group $\left(F(1,38)=6.43 ; p=0.01 ; \eta_{P}^{2}=0.14\right)$ and a time x group interaction $\left(F(2,75.99)=18.60 ; p<0.001 ; \eta_{P}^{2}=0.32\right)$. Post-hoc tests revealed that, compared to the control group, the stress group reported significantly higher pain ratings at the second time point (i.e., after the stress challenge; $p<0.0001$; two tailed). Similarly, the subjective ratings of unpleasant feelings were significantly higher in the stress group, compared to the control group (main 
effect of time $F(1.88,71.64)=7.46 ; p=0.001 ; \eta_{\mathrm{P}}^{2}=0.16$, main effect of group $F(1,38)=9.65 ; p=$ $0.004 ; \eta_{\mathrm{P}}^{2}=0.20$, time $\mathrm{x}$ group interaction, $\left.F(1.88,71.64)=11.31 ; p<0.0001 ; \eta_{\mathrm{P}}{ }^{2}=0.23\right)$. Pair-wise between-groups comparisons showed that this difference was significant at the second time point after the stressor $(p<0.001$; two-tailed). Fig. 3 shows a graphical description of these results.

Figure 3

Fig 3. The stress group reported significantly higher levels of pain and unpleasant feelings than the control group at $\mathrm{T} 2$ after the stressor. Plots represent mean scores of the subjective ratings of stress, pain, and unpleasant feelings for the Stress and Control groups at Time 0 (baseline), T2 (after the Socially Evaluated Cold Pressor Test - SECPT or Warm Water Test - WWT) and T5 (after the Inequality Game - IG). ${ }^{*} p<0.001$; two-tailed. Bars represent 2 standard errors of the mean (s.e.m, one above and one below the mean).

The stress induction increased the levels of cortisol, but no evidence was found for an increase of the activity of alpha-amylase

The resulting values of cortisol and alpha-amylase were log-transformed before the statistical analysis in order to approximate a normal distribution. To test for changes of salivary cortisol and alpha-amylase levels in both groups across the experimental session, we conducted a repeated measures ANOVA with "time" (8 time points) as a within-subject factor and "group" (stress and control) as a between-subject factor. As shown in Fig. 4A, the stressor affected cortisol levels differently across time in the stress vs. control condition (significant time $\mathrm{x}$ group interaction $\left(F(3.04,115.86)=2.88 ; p=0.03, \eta_{\mathrm{P}}^{2}=0.07\right)$. There was also a significant main effect of time $\left(F(3.04,115.86)=28.63 ; p<0.001, \eta_{\mathrm{P}}^{2}=0.43\right)$, but no significant effect of group $(F(1,38)=2.26 ; p=0.14)$. Replicating previous findings $(47)$, in the stress group, cortisol levels peaked approximately 20 minutes after cessation of the stressor (T3, before the IG vs. T2, after the stress cessation $p=0.02$ ). In contrast, the control group had significant decreases of salivary cortisol levels between T2 (after the stress cessation) and T3 (before the IG; $p=0.01$ ). Crucially, the stressor-induced peak cortisol levels were significantly higher in the stress vs. the control group (T3, 
before starting the IG $(p=0.007)$. Cortisol levels in the stress group then started to decrease during the first phase of the IG (T3, before the IG vs. T4, between phase A and B of the IG; $p<0.001$ ). The activity of alpha-amylase was not statistically significant different between the eight sampling time points, nor between groups (time x group interaction $F(4.14,149.38)=1.70 ; p=0.74$, main effect of time $F(4.14$, $149.38)=0.49 ; p=0.15$, main effect of group $F(1,36)=0.40 ; p=0.49)$. Fig. 4B.

Figure 4A \& 4B

Fig. 4A and 4B. The plots depict true (non-logarithmic) means: A Mean cortisol levels (nmol/l) and B mean alpha-amylase activity (U/ml) across the experimental session for the stress and control groups. The timing of the stressor (SECPT) or control induction (WWT) and the timing of the Inequality Game (IG) are shown in the figure. ${ }^{*} p<0.05 ; * p=0.007$. Bars represent 2 standard errors of the mean (s.e.m, one above and one below the mean).

\section{Stress induction increases punishment and decreases cooperation in the Inequality Game}

To test whether the stress induction had an effect on cooperative (coded as positive values) or competitive (coded as negative values) behavior during the second phase of the IG (high power for the participant), we conducted a repeated measures ANOVA with "other player" (economic behavior towards the fair or unfair other player) as a within-subject factor and "group" (stress vs. control) as a between-subject factor. As shown in Fig. 5, we observed a main effect of other player $(F(1,38)=15.09$; $\left.p<0.001 ; \eta \mathrm{P}^{2}=0.28\right)$, a marginally significant effect of $\operatorname{group}\left(F(1,38)=3.92 ; p=0.05 ; \eta_{\mathrm{P}}{ }^{2}=0.09\right)$, and a group $\mathrm{x}$ other player interaction $\left(F(1,38)=4.52 ; p=0.04 ; \eta_{\mathrm{P}}^{2}=0.11\right)$. Confirming previous findings $(33,45)$, participants across both groups had a more cooperative economic behavior towards the fair as opposed to the unfair other player. Follow up pairwise t-test revealed that this difference was more pronounced in the stress group $(t(18)=3.26 ; p=0.004)$ and was only a trend in the control group $(t(20)=1.92 ; p=0.07)$. Most importantly, independent $\mathrm{t}$-tests showed that, whereas there was no significant difference in the economic choices for the fair other player between the stress group and the control group $(t(38)=0.95 ; p=0.35)$, the stress group showed a more competitive behavior towards the unfair other player than the control group $(t(38)=2.66 ; p=0.01)$. Furthermore, we observed a 
significant positive correlation between the cooperative behavior towards the fair and unfair other player in the control group (Spearman's $\rho=0.80, p<0.001$ ), but not in the stress group (Spearman's $\rho=0.25$, $p=0.28$ ), resulting in a significant difference between the two correlation coefficients (Fischer's $z=$ $2.45, p=0.01$ two-tailed (48)).

Figure 5

Fig 5. Participants in the stress group punished the unfair other more than participants in the control group. Charts depict mean cooperative (positive values) minus competitive (negative values) economic behavior in the Inequality Game towards the fair and unfair other in the stress and control groups. Between-group tests showed a more competitive behavior towards the unfair other between the control and stress group $\left({ }^{*} p=0.01\right)$. Pair-wise comparisons showed significant differences in behavior towards the fair compared to the unfair other in the stress group $(* * p=0.004)$. Bars represent 2 standard errors of the mean (s.e.m, one above and one below the mean).

Lastly, we classified participants as prosocial, sanctioning or competitive ( $n=14, n=1, n=5$ in the control group and $n=5, n=7, n=7$ in the stress group, respectively) according to their choices towards both players (see Supplemental Material 1). Replicating previous findings (33), binomial tests showed that participants behaved more prosocially than we would expect by chance distribution in the control group $(p<0.001)$, but not in the stress group $(p=0.99)$. Fischer's Exact Test for comparing the binomial proportions between both groups showed that the number of prosocial participants was significantly higher in the control vs. stress group $(p=0.01)$, while the number of sanctioning participants was significantly higher in the stress vs. control group $(p=0.01)$. Conversely, the proportion of participants classified as competitive was not significantly different between groups ( $p=0.44$; Fig. 6$)$.

Fig. 6. More participants were classified as "Sanctioning" and less participants were classified as "Prosocial" in the stress group. Frequency plots depict the classification of the participants based on 
their economic choices for the control and stress groups. Participants were classified as "Prosocial" (i.e., cooperative with both the fair and unfair other), "Sanctioning" (i.e., cooperative with the fair other and competitive with the unfair other) or "Competitive" (i.e., competitive with both the fair and unfair other). Fisher's Exact Test showed significant differences in distributions between groups for the "Prosocial" (14 vs. 5 in control and stress groups respectively; $p=0.01$ ) and "Sanctioning" ( 1 vs. 7 in control and stress group respectively; $p=0.01$ ).

Next, we investigated whether differences between groups observed in the IRI-Empathic Concern scale explained the behavior of our participants. To test this, we conducted an analysis of covariance (ANCOVA), with Empathic Concern as a covariate in our repeated measures model. This analysis showed that, when controlling for the levels of Empathic Concern among participants, the main effect of group remained significant $\left(F(1,37)=5.64 ; p=0.02 ; \eta_{\mathrm{P}}^{2}=0.13\right)$, the main effect of other player was not significant anymore $\left(F(1,37)=0.17 ; p=0.67 ; \eta_{\mathrm{P}}^{2}=0.0\right)$ and the group $\mathrm{x}$ other interaction became a trend $\left(F(1,37)=3.60 ; p=0.06 ; \eta_{\mathrm{P}}^{2}=0.09\right)$. Importantly, independent t-tests revealed that, after controlling for Empathic Concern, the difference in behavior for the fair other player remained nonsignificant between both groups $(t(38)=1.24 ; p=0.22)$, while the difference in behavior for the unfair other was still significantly higher in the stress group compared to the control group $(t(38)=2.64 ; p=$ 0.01). On the other hand, after controlling for Empathic Concern, the difference in behavior towards both players became not significant in both groups (all $p s>0.15$. Further details are provided in Supplemental Material 2 and Fig. S1).

Finally, we tested whether the stress induction had an effect on the feedback sent to the fair and unfair other. Repeated measures ANOVA showed a trend towards a significant effect of other player $(F(1,38)$ $\left.=3.78 ; p=0.06 ; \eta_{\mathrm{P}}^{2}=0.09\right)$, a trend for an effect of group $\left(F(1,38)=3.00 ; p=0.09 ; \eta_{\mathrm{P}}^{2}=0.07\right)$, and no group $\mathrm{x}$ other player interaction $\left(F(1,38)=0.15 ; p=0.70 ; \eta_{\mathrm{P}}^{2}=0.0\right)$. Overall, participants had a tendency to give more positive feedback to the fair than unfair other player and the stress group showed a tendency to give less positive feedback to both players than the control group (see Supplemental Fig. S2). 


\section{Relationship between cortisol levels and behavior towards the unfair other in the Inequality}

\section{Game}

357 Finally, we tested whether the levels of cortisol during the IG were associated with the observed 358 punishment behavior towards the unfair other player in the stress group: We computed the area under 359 the curve with respect to ground $\left(\mathrm{AUC}_{\mathrm{G}}\right)$ to obtain a single value of salivary cortisol output and then correlated $\mathrm{AUC}_{\mathrm{G}}$ values with behavior towards the unfair other player (49). (See Supplemental Material

$361 \mathrm{~S} 4$ for a description of the computation of the $\mathrm{AUC}_{\mathrm{G}}$ ). Rank correlations revealed a negative relationship 362 between the $\mathrm{AUC}_{\mathrm{G}}$ and the behavior towards the unfair other (Spearman's $\rho=-0.48, p=0.03$; Fig 7), indicating that higher levels of cortisol were associated with more punishing behavior (coded as negative values) towards the unfair player. Similar results were obtained in a second correlation analysis between a time-specific $\mathrm{AUC}_{\mathrm{G}}$ (only the time points that corresponded to the IG: $\mathrm{T} 4$ - T6) and the punishment behavior towards the unfair other (Spearman's $\rho=-0.51, p=0.02$ ). In summary, both analyses suggest that higher cortisol levels in response to the stress induction procedure are related to the degree of punishment behavior towards the unfair other.

Fig. 7. Higher cortisol levels in the stress group predict less cooperative behavior (i.e., more punishment) towards the unfair other in the Inequality Game. Scatter plot depicts the relationship between the economic behavior towards the unfair other (cooperative minus competitive) and the total area under the Curve with respect to Ground $\left(\mathrm{AUC}_{\mathrm{G}}\right)$ in the stress group. 


\section{Discussion}

375 This controlled study investigated whether experimentally induced stress increases aggressive and

376

377

378

379

380

381

382

383

384

385

386

387 punishment behaviors during economic interactions. In agreement with previous studies, peak cortisol levels occurred approximately 20 minutes after the stress cessation (47), thereby confirming the effectiveness of the stress induction. The present results support the hypothesis that stress increases punishment behavior, which was operationalized as competitive behavior toward an unfair player during the Inequality Game (IG). The difference in behavior towards the unfair player between the stress and control group remained significant after controlling for inter-individual differences in empathy scores at baseline (i.e., IRI- Empathic Concern). There were no significant differences in competitive behavior towards the fair other between groups, indicating that the stress induction did not trigger more aggression. Moreover, we observed the uncoupling of the cooperative behavior towards the fair and unfair other in the stressed group. More specifically, in the stress group participants who behaved more cooperatively towards the fair other, did not behave in a more cooperative way towards the unfair other. In addition, compared to the control group, more participants from the stress group showed sanctioning behavior and less participants from the same group showed prosocial behavior. Finally, higher cortisol levels in the stress group were associated with more punishment behavior, as evidenced by a linear negative relationship between overall cortisol output and cooperative behavior towards the unfair other. Interestingly, despite the rise in cortisol levels, self-reported measures of stress were not significantly different between groups. By including behavioral and biological measures, our study extends findings of previous longitudinal studies that reported a link between early life stress and later hostility during social interactions, and highlights the role of increased cortisol levels as a potential facilitator of active punishment in anger-provoking situations.

The present study demonstrated that inducing stress results in increased punishment behavior towards unfair opponents. If we consider that the legitimacy of punishment is often taken as a first step in the escalation towards aggressive encounters (50), the presence of stressors might increase the propensity to escalate interpersonal or even intergroup conflicts. Previous experiments have shown, for instance, that higher self-reported stress while driving to work is associated with subsequent reports of anger and 
hostility at the workplace (14) and that punishment behaviors are exhibited more frequently after an experimental stress induction (31). The present study extends this research by showing that the degree of punishment is related to the cortisol increase from the stress induction - thus suggesting a critical role of cortisol for punishment behavior. A similar link between naturally occurring cortisol variations and aggression has been described, for instance, in participants who behaved aggressively playing the Taylor Aggression Paradigm (51) and adolescents with an aggressive form of conduct disorder (52). Although these results may be particularly important for understanding pathological impulsivity, they also explain how cortisol facilitates aggressive behaviors in healthy individuals who face unfair situations. Thus, future work is needed to disentangle the immediate and long-term consequences of stressful situations in social contexts, as well as to identify inter-individual factors that may prevent the escalation of stressrelated aggression.

We also observed that in the stress group, more individuals were classified as "Sanctioning" and less individuals were classified as "Prosocial", compared to the control group. In the same line, the positive relationship between cooperative behavior towards fair and unfair others in the control group, was not present in the stress group, indicating that reactions to provocations in the form of punishment became more pronounced with stress and more independent of general tendencies for cooperative or competitive behavior. While several studies support our results by showing that stress reduces prosocial behaviors $(18,53)$, other studies have shown that experimentally induced stress can trigger altruistic decisions or even promote sharing behaviors $(16,17)$. Even when the underlying mechanisms of these contradictory outcomes are yet to be understood, the sex of the participants (i.e., females (15)) and the group membership of the confederates seems to play a role in the positive relationship between stress and prosocial behaviors $(18,54,55)$. In line with these findings, our study suggests that stress-related punishment can be a motivational factor that contributes to increase or maintain intergroup conflicts, especially among males. Thus, it will be particularly interesting to further test the impact of both, stress or stress reduction, on intergroup conflicts.

As previously described (47), the stress induction resulted in higher subjective ratings of pain and unpleasant feelings. However, the subjective ratings of stress decreased across the experimental session 
in participants from both, the control and stress group. Similar inconsistencies between self-reported and physiological measures of stress have been previously described and may arise from a weak construct validity of the Visual Analogue Scale (56) or the rather small magnitude of the endocrine response of experimentally induced stress (57), which might not be comparable with the classical definition of stress among our participants (e.g., considered an alarm response (22)). Another potential explanation for this inconsistency is given by selection bias in self-report measures of stress due to a higher gender-related desirability (i.e., masculinity related to less emotionality (58)). Hence, our study cannot conclude that the observed effects are caused by the conscious experience of stress, but instead are driven by cortisol increases elicited by the stress induction.

In summary, our results extend previous findings by showing that a stress induction in a randomized controlled laboratory experiment not only increased punishment behavior, but that the increase in punishment was linearly related to the increase in stress-induced cortisol levels, thus suggesting a direct link between biological stress markers and the presentation of punishment behavior. Further research should expand our findings by investigating whether the stress induction creates incidental negative emotions that prepare the organism to appraise the following situation as unfair (59) and therefore, increase aggressive or punishment responses.

Our study has several limitations. First, we acknowledge the modest sample size of this experiment and recognize that this factor may have played a role, for instance, in the behavioral differences for the fair and unfair other being only at trend level in the control group. Hence it is likely that we were able to detect relatively large effect sizes, as shown by our sensitivity analyses (with a power of $1-\beta=0.80$, resulting $\alpha=0.004, \mathrm{~d} z=0.88(60))$ and potentially, omit small effects when comparing behavioral responses within groups. Another limitation derived from the sample size is the significant difference observed in the IRI- Empathic Concern between our experimental groups, with a higher mean of the control group. While we observed that the difference in empathic scores explained some variance in our analyses, empathic concern scores did not affect the difference in behavior towards the unfair other between groups, highlighting the role of biological stress in provoking situations. Finally, due to technical problems at the time of data acquisition, we could not acquire measures of autonomic arousal, 
as initially planned (i.e., skin conductance response and heart rates). These measures would have allowed us to make stronger claims on the interaction between the hypothalamic-pituitary-adrenal axis (HPA) and the catecholaminergic system in stress-induced punishment or aggression. Even though the interplay between both systems was previously described $(23,29,55,61)$, the lack of these measures and the non-significant results in the levels of alpha-amylase (taken as a marker of adrenergic activity (37)), raise caution in inferring a direct relationship between cortisol and arousal from our results.

Taken together, the findings of this experiment suggest that, even in cases when stress is not reported as such by participants (who still report pain and unpleasantness), social and physical stressors could predispose individuals to punish unfair others. Furthermore, our results suggest that this punishment behavior is related to higher stress-induced cortisol levels, showing the implication of neuroendocrine responses in social behaviors. The present results provide further insights into the impact of stress induction on interpersonal and inter-group interactions and on its biological underpinnings. The awareness of the detrimental impact of stress on social behavior may increase the efforts to reduce stressors in a variety of settings, including families, schools, work-places, or negotiations.

\section{Acknowledgments}

We thank Emna El May and Kacylia Pistoia for their help in collecting the data. This study was conducted using the facilities of the Brain and Behaviour Laboratory (BBL) of the University of Geneva and benefited from support of the BBL technical staff. Y.I.D.A is supported by the Medit-Ageing project, funded through the European Union's Horizon 2020 Research and Innovation Program (grant 667696). This work was supported by the National Center of Competence in Research (NCCR) for the Affective Sciences, financed by a grant from the Swiss National Science Foundation (51NF40- 104897), hosted by the University of Geneva. U.R. was supported by the Pierre Mercier Foundation and the Swiss National Science Foundation (PCEFP1_186911).

\section{Author contributions}

477 Conceptualization: LG, DS, UR and OMK; Data curation: LG and CA; Formal analysis: YIDA, CA and 478 OMK; Funding acquisition: DS; Methodology: YIDA, UR, DS and OMK; Project administration: 
479 OMK, DS, UR; Supervision: DS, UR and OMK; Visualization: YIDA and OMK; Writing: YIDA and

480 OMK; Writing - review \& editing: all authors. All authors approved the final version of the manuscript.

\section{Competing interests}

481 The authors declare no conflicts of interest.

\section{Data availability}

482 The data underlying this study are available at Open Science Framework. A database with scores and 483 measures, calculations and plotting scripts can be accessed via https://osf.io/qbvgu/. 


\section{References}

484

485

486

487

488

489

490

491

492

493

494

495

496

497

498

499

500

501

502

503

504

505

506

507

508

509

510

511

512

513

514

515

516

517

518

519

520

521

522

523

524

525

526

527

528

529

530

531

532

533

1. Tyson PD. Physiological arousal, reactive aggression, and the induction of an incompatible relaxation response. Aggression and Violent Behavior. 1998;3(2):143-58.

2. Thomas AL, Davis SM, Dierick HA. Of Fighting Flies, Mice, and Men: Are Some of the Molecular and Neuronal Mechanisms of Aggression Universal in the Animal Kingdom? PLoS Genet. 2015;11(8):e1005416.

3. Church RM. The Varied Effects of Punishment on Behavior. Psychol Rev. 1963;70:369-402. 4. de Quervain DJ, Fischbacher U, Treyer V, Schellhammer M, Schnyder U, Buck A, et al. The neural basis of altruistic punishment. Science. 2004;305(5688):1254-8.

5. Gollwitzer M, Meder M, Schmitt $M$. What gives victims satisfaction when they seek revenge? European Journal of Social Psychology. 2011;41(3):364-74.

6. Nelson RJ, Trainor BC. Neural mechanisms of aggression. Nat Rev Neurosci. 2007;8(7):536-46. 7. Reiss AJ, Roth JA, Miczek KA, National Research Council (U.S.). Panel on the Understanding and Control of Violent Behavior. Understanding and preventing violence. Washington, D.C.: National Academy Press; 1993.

8. Tuvblad C, Baker LA. Human aggression across the lifespan: genetic propensities and environmental moderators. Adv Genet. 2011;75:171-214.

9. Abbink K, Gangadharan L, Handfield T, Thrasher J. Peer punishment promotes enforcement of bad social norms. Nature Communications. 2017;8.

10. Wilkinson R. Why is violence more common where inequality is greater? Ann N Y Acad Sci. 2004;1036:1-12.

11. Bolton JL, Molet J, Ivy A, Baram TZ. New insights into early-life stress and behavioral outcomes. Curr Opin Behav Sci. 2017;14:133-9.

12. Haller J, Mikics E, Halasz J, Toth M. Mechanisms differentiating normal from abnormal aggression: glucocorticoids and serotonin. Eur J Pharmacol. 2005;526(1-3):89-100.

13. Scarpa A, Raine A. Psychophysiology of anger and violent behavior. Psychiatric Clinics of North America. 1997;20(2):375-\&.

14. Hennessy DA. The impact of commuter stress on workplace aggression. Journal of Applied Social Psychology. 2008;38(9):2315-35.

15. Taylor SE, Klein LC, Lewis BP, Gruenewald TL, Gurung RA, Updegraff JA. Biobehavioral responses to stress in females: tend-and-befriend, not fight-or-flight. Psychol Rev. 2000;107(3):41129.

16. von Dawans B, Fischbacher U, Kirschbaum C, Fehr E, Heinrichs M. The social dimension of stress reactivity: acute stress increases prosocial behavior in humans. Psychol Sci. 2012;23(6):651-60. 17. Singer N, Sommer M, Dohnel K, Zankert S, Wust S, Kudielka BM. Acute psychosocial stress and everyday moral decision-making in young healthy men: The impact of cortisol. Hormones and Behavior. 2017;93:72-81.

18. Steinbeis $N$, Engert $V$, Linz $R$, Singer $T$. The effects of stress and affiliation on social decisionmaking: Investigating the tend-and-befriend pattern. Psychoneuroendocrinology. 2015;62:138-48. 19. Lazarus RS, Folkman S. Stress, Appraisal, and Coping. New York : Springer Pub Co. 1984. 20. Pool ER, Sander D. Vulnerability to relapse under stress: insights from affective neuroscience. Swiss Med Wkly. 2019;149:w20151.

21. Dickerson SS, Kemeny ME. Acute stressors and cortisol responses: a theoretical integration and synthesis of laboratory research. Psychol Bull. 2004;130(3):355-91.

22. Ursin H, Eriksen HR. The cognitive activation theory of stress. Psychoneuroendocrinology. 2004;29(5):567-92.

23. Haller J, Makara GB, Kruk MR. Catecholaminergic involvement in the control of aggression: hormones, the peripheral sympathetic, and central noradrenergic systems. Neurosci Biobehav Rev. 1998;22(1):85-97.

24. Arnsten AF. Stress weakens prefrontal networks: molecular insults to higher cognition. Nat Neurosci. 2015;18(10):1376-85. 
25. Volkow ND, Tancredi LR, Grant C, Gillespie H, Valentine A, Mullani N, et al. Brain glucose metabolism in violent psychiatric patients: a preliminary study. Psychiatry Res. 1995;61(4):243-53. 26. Marquez C, Poirier GL, Cordero MI, Larsen MH, Groner A, Marquis J, et al. Peripuberty stress leads to abnormal aggression, altered amygdala and orbitofrontal reactivity and increased prefrontal MAOA gene expression. Transl Psychiatry. 2013;3:e216.

27. Sandi C, Haller J. Stress and the social brain: behavioural effects and neurobiological mechanisms. Nat Rev Neurosci. 2015;16(5):290-304.

28. Sprague J, Verona E, Kalkhoff W, Kilmer A. Moderators and Mediators of the StressAggression Relationship: Executive Function and State Anger. Emotion. 2011;11(1):61-73. 29. Vogel S, Schwabe L. Stress, aggression, and the balance of approach and avoidance. Psychoneuroendocrinology. 2019;103:137-46.

30. Clutton-Brock TH, Parker GA. Punishment in animal societies. Nature. 1995;373(6511):20916.

31. Vinkers CH, Zorn JV, Cornelisse S, Koot S, Houtepen LC, Olivier B, et al. Time-dependent changes in altruistic punishment following stress. Psychoneuroendocrinology. 2013;38(9):1467-75. 32. Schwabe L, Haddad L, Schachinger H. HPA axis activation by a socially evaluated cold-pressor test. Psychoneuroendocrinology. 2008;33(6):890-5.

33. Klimecki OM, Vuilleumier $P$, Sander D. The Impact of Emotions and Empathy-Related Traits on Punishment Behavior: Introduction and Validation of the Inequality Game. PLoS One. 2016;11(3):e0151028.

34. Bettencourt BA, Miller N. Gender differences in aggression as a function of provocation: a meta-analysis. Psychol Bull. 1996;119(3):422-47.

35. Bangasser DA, Wiersielis KR. Sex differences in stress responses: a critical role for corticotropin-releasing factor. Hormones (Athens). 2018;17(1):5-13.

36. Fries E, Dettenborn L, Kirschbaum C. The cortisol awakening response (CAR): facts and future directions. Int J Psychophysiol. 2009;72(1):67-73.

37. Nater UM, Rohleder N, Schlotz W, Ehlert U, Kirschbaum C. Determinants of the diurnal course of salivary alpha-amylase. Psychoneuroendocrinology. 2007;32(4):392-401.

38. Lorentz K, Gutschow B, Renner F. Evaluation of a direct alpha-amylase assay using 2-chloro-4nitrophenyl-alpha-D-maltotrioside. Clin Chem Lab Med. 1999;37(11-12):1053-62.

39. Davis $\mathrm{MH}$. Measuring Individual-Differences in Empathy - Evidence for a Multidimensional Approach. Journal of Personality and Social Psychology. 1983;44(1):113-26.

40. Gilet AL, Mella N, Studer J, Gruhn D, Labouvie-Vief G. Assessing Dispositional Empathy in Adults: A French Validation of the Interpersonal Reactivity Index (IRI). Canadian Journal of Behavioural Science-Revue Canadienne Des Sciences Du Comportement. 2013;45(1):42-8.

41. Watson D, Clark LA, Tellegen A. Development and validation of brief measures of positive and negative affect: the PANAS scales. J Pers Soc Psychol. 1988;54(6):1063-70.

42. Gaudreau P, Sanchez X, Blondin JP. Positive and negative affective states in a performancerelated setting - Testing the factorial structure of the PANAS across two samples of French-Canadian participants. European Journal of Psychological Assessment. 2006;22(4):240-9.

43. Smeets T, Cornelisse S, Quaedflieg CWEM, Meyer T, Jelicic M, Merckelbach H. Introducing the Maastricht Acute Stress Test (MAST): A quick and non-invasive approach to elicit robust autonomic and glucocorticoid stress responses. Psychoneuroendocrinology. 2012;37(12):1998-2008.

44. Pool E, Brosch T, Delplanque S, Sander D. Stress increases cue-triggered "wanting" for sweet reward in humans. J Exp Psychol Anim Learn Cogn. 2015;41(2):128-36.

45. Klimecki OM, Sander D, Vuilleumier P. Distinct Brain Areas involved in Anger versus Punishment during Social Interactions. Sci Rep. 2018;8(1):10556.

46. Taylor SP. Aggressive behavior and physiological arousal as a function of provocation and the tendency to inhibit aggression. J Pers. 1967;35(2):297-310.

47. Schwabe L, Schachinger H. Ten years of research with the Socially Evaluated Cold Pressor Test: Data from the past and guidelines for the future. Psychoneuroendocrinology. 2018;92:155-61. 
48. Diedenhofen B, Musch J. cocor: a comprehensive solution for the statistical comparison of correlations. PLoS One. 2015;10(3):e0121945.

49. Pruessner JC, Kirschbaum C, Meinlschmid G, Hellhammer DH. Two formulas for computation of the area under the curve represent measures of total hormone concentration versus timedependent change. Psychoneuroendocrinology. 2003;28(7):916-31.

50. Mummendey Al. Social psychology of aggression : from individual behavior to social interaction. Berlin ; New York: Springer-Verlag; 1984. xiv, 176 p. p.

51. Bohnke R, Bertsch K, Kruk MR, Naumann E. The relationship between basal and acute HPA axis activity and aggressive behavior in adults. J Neural Transm (Vienna). 2010;117(5):629-37. 52. van Bokhoven I, Van Goozen SH, van Engeland H, Schaal B, Arseneault L, Seguin JR, et al. Salivary cortisol and aggression in a population-based longitudinal study of adolescent males. J Neural Transm (Vienna). 2005;112(8):1083-96.

53. Starcke K, Polzer C, Wolf OT, Brand M. Does stress alter everyday moral decision-making? Psychoneuroendocrinology. 2011;36(2):210-9.

54. von Dawans B, Trueg A, Kirschbaum C, Fischbacher U, Heinrichs M. Acute social and physical stress interact to influence social behavior: The role of social anxiety. PLoS One. 2018;13(10):e0204665.

55. Schweda A, Faber NS, Crockett MJ, Kalenscher T. The effects of psychosocial stress on intergroup resource allocation. Sci Rep. 2019;9(1):18620.

56. Campbell J, Ehlert U. Acute psychosocial stress: does the emotional stress response correspond with physiological responses? Psychoneuroendocrinology. 2012;37(8):1111-34.

57. Stalder T, Steudte-Schmiedgen S, Alexander N, Klucken T, Vater A, Wichmann S, et al. Stressrelated and basic determinants of hair cortisol in humans: A meta-analysis.

Psychoneuroendocrinology. 2017;77:261-74.

58. Mayor E. Gender roles and traits in stress and health. Front Psychol. 2015;6:779.

59. Lerner JS, Keltner D. Beyond valence: Toward a model of emotion-specific influences on judgement and choice. Cognition \& Emotion. 2000;14(4):473-93.

60. Faul F, Erdfelder E, Buchner A, Lang AG. Statistical power analyses using G*Power 3.1: tests for correlation and regression analyses. Behav Res Methods. 2009;41(4):1149-60.

61. Krugers $\mathrm{HJ}$, Karst $\mathrm{H}$, Joels $\mathrm{M}$. Interactions between noradrenaline and corticosteroids in the brain: from electrical activity to cognitive performance. Front Cell Neurosci. 2012;6:15. 


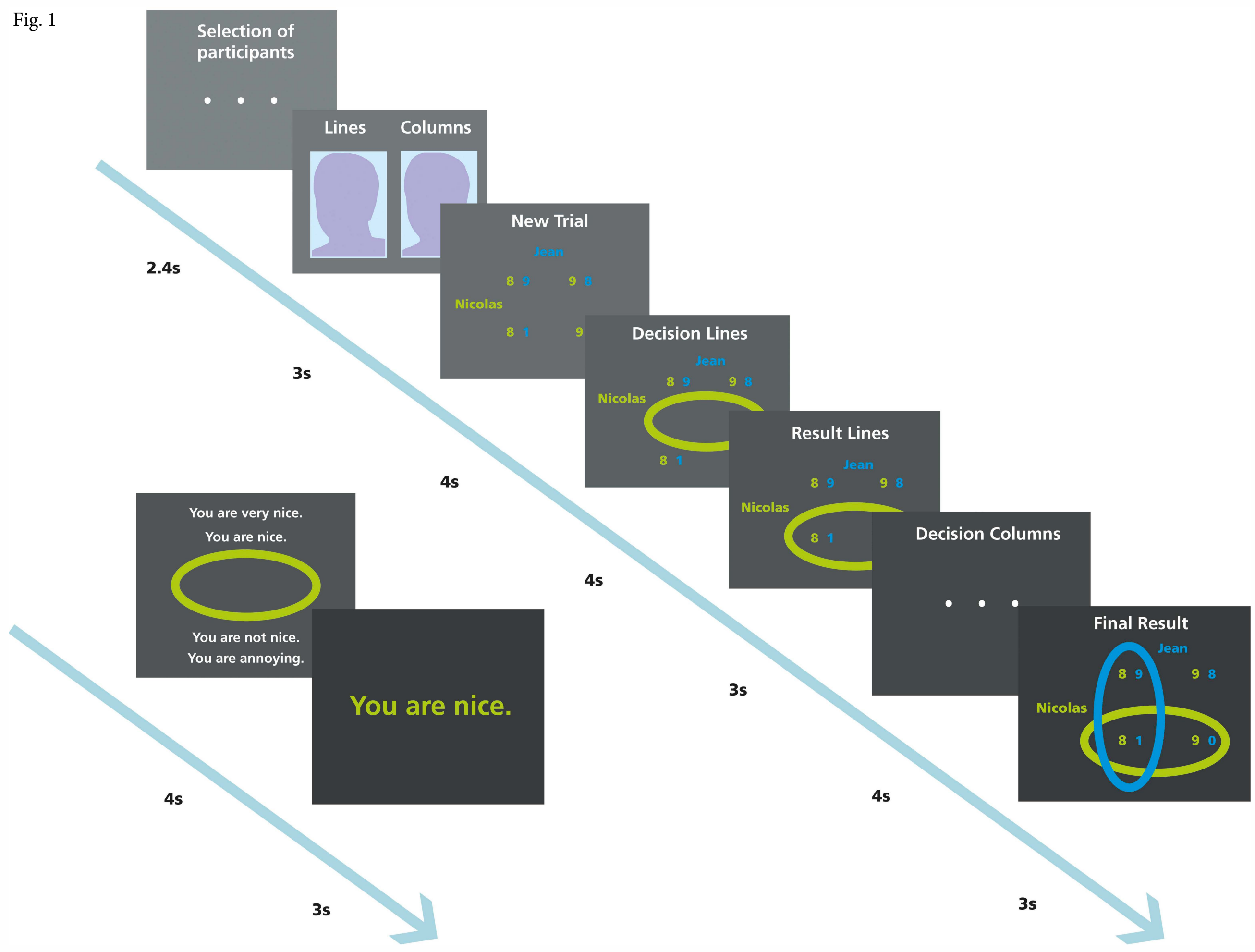


Fig. 2

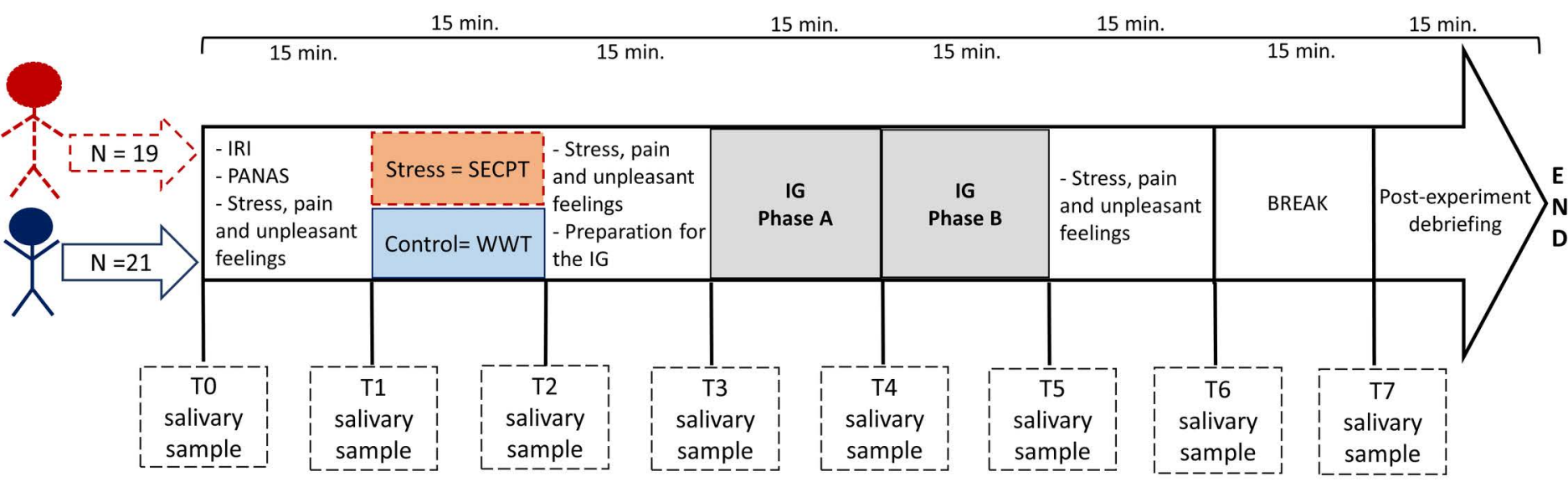


Fig. 3

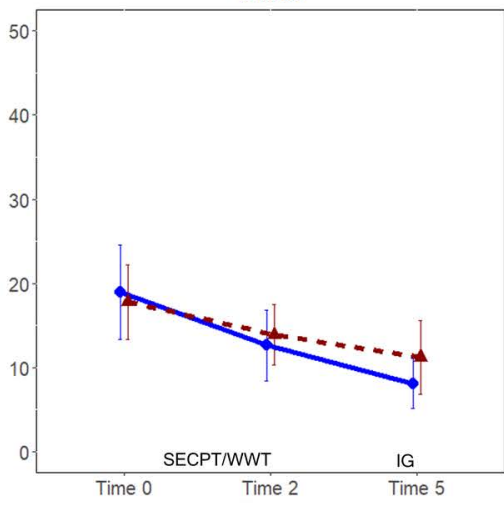

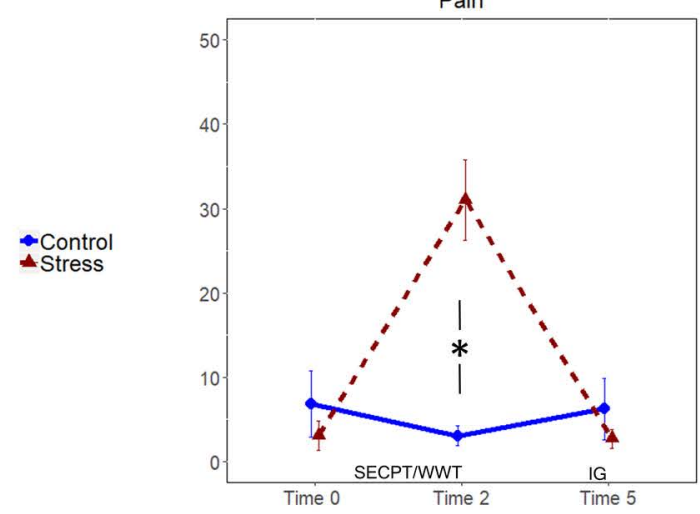

- Control

- Stress

Unpleasant Feelings

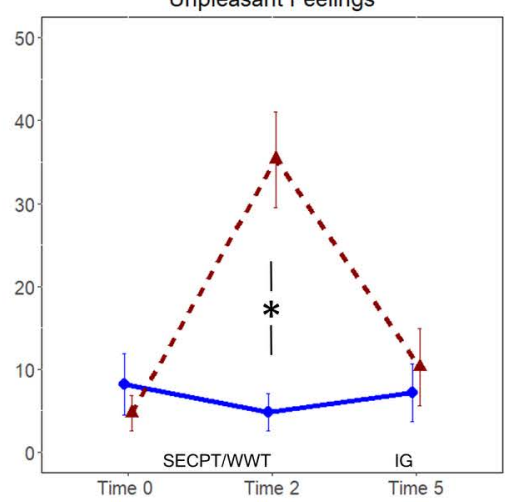

- Control

$\star$ Stress 
Fig. 4

A

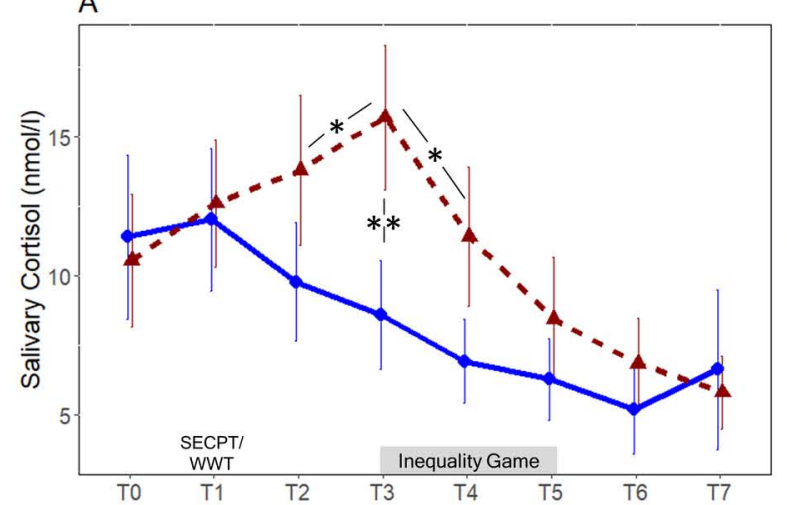

- Control

- Stress

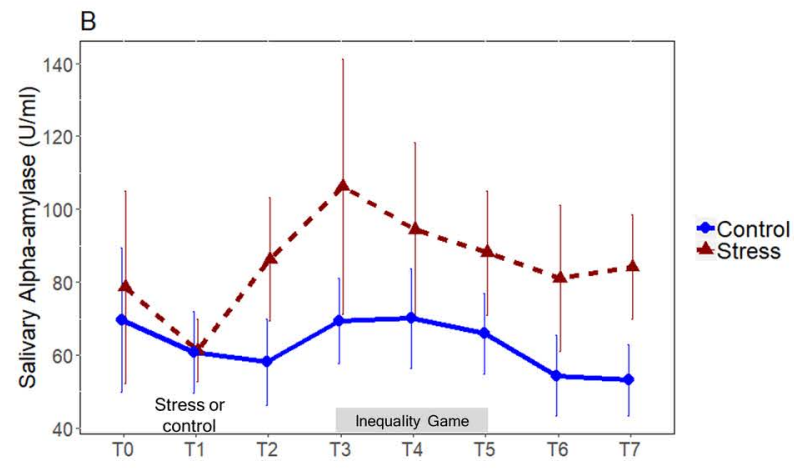


Fig. 5

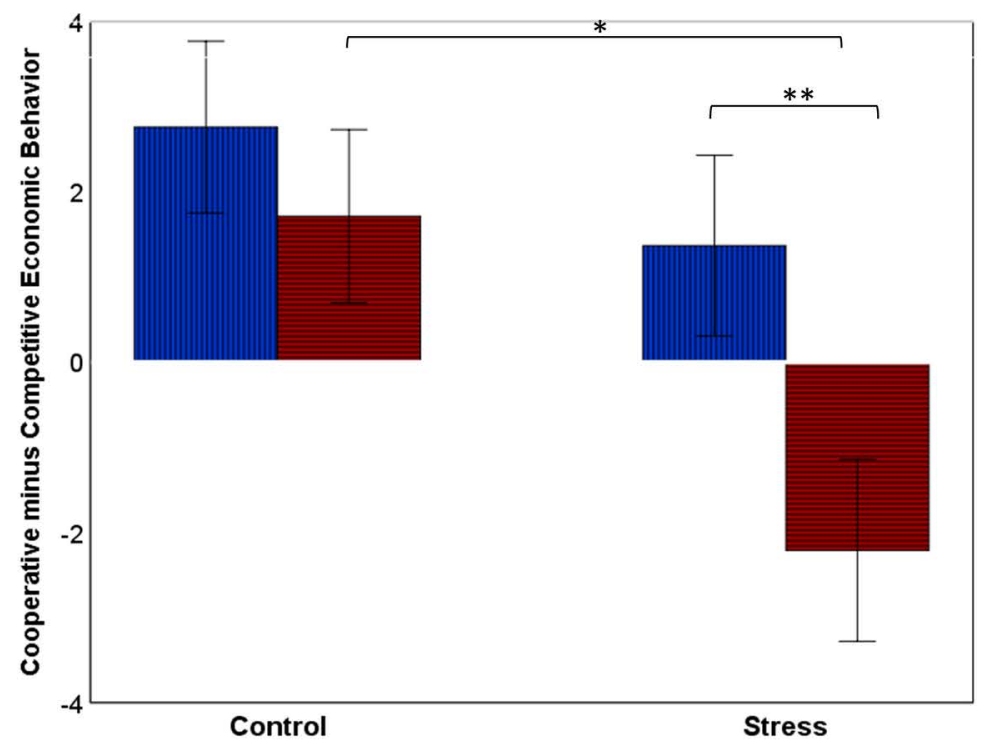

四Fair other

目Unfair other 
Fig. 6

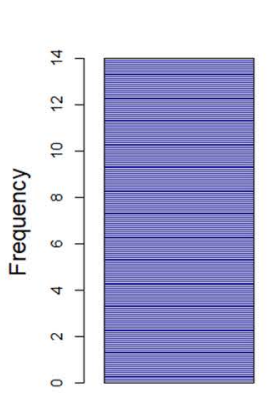

Prosocial
CONTROL

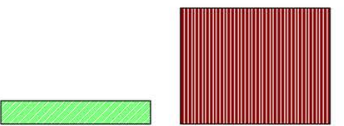

Sanctioning

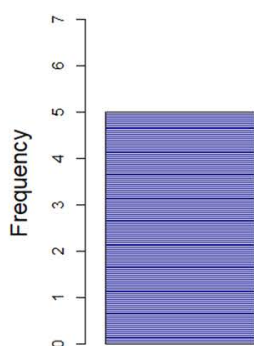

Prosocial
STRESS

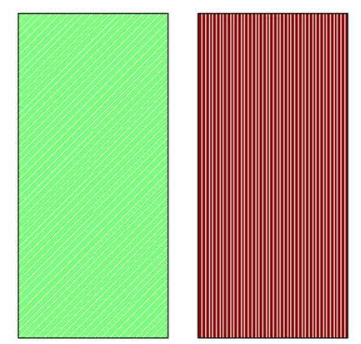

Sanctioning

Competitive 
Fig. 7
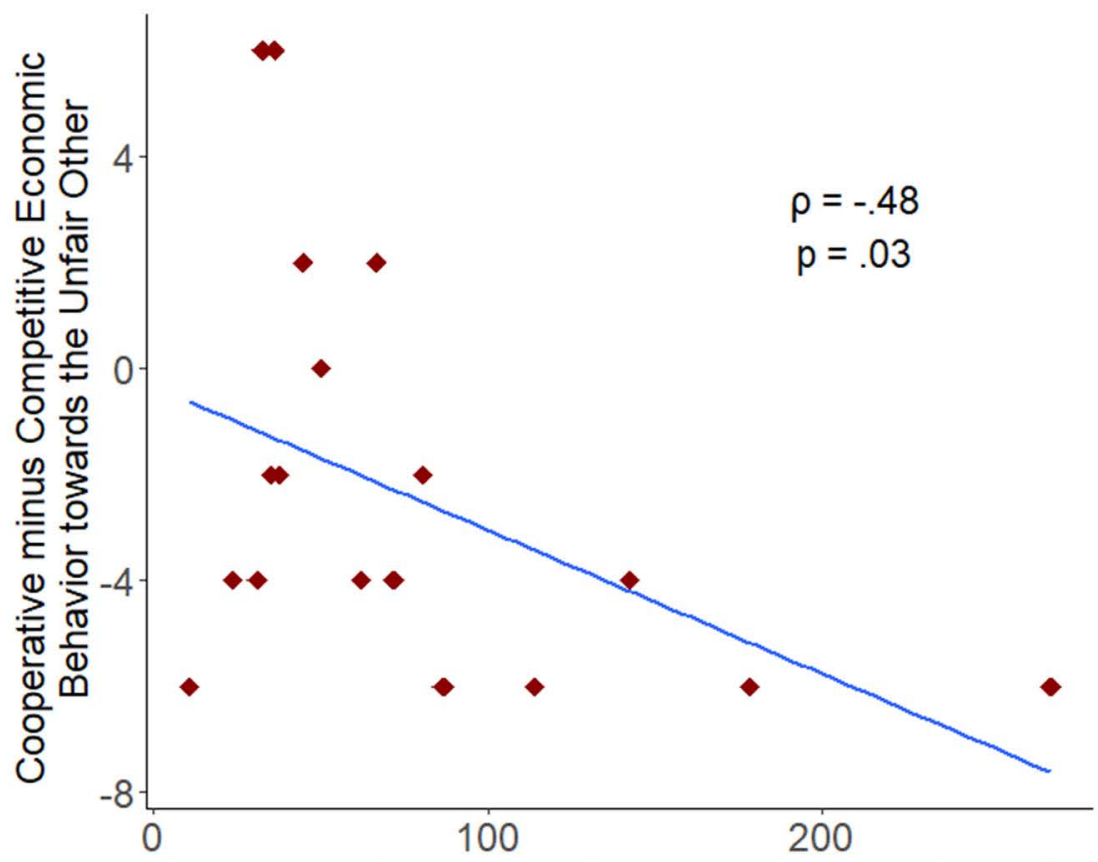

Area under the curve with respect to ground - $\mathrm{AUC}_{\mathrm{G}}$ 